Silva, Solange Cristina da; Souza, Márcio Vieira de;

"DESENHO UNIVERSAL PARA APRENDIZAGEM E MOOCS:

UMA REFLEXÃO PRELIMINAR", p. 129-138 . In: Souza,

Márcio Vieira de; Giglio, Kamil. Mídias Digitais, Redes

Sociais e Educação em Rede: Experiências na

Pesquisa e Extensão Universitária. São Paulo: Blucher,

2015. ISBN: 978-85-8039-128-2, DOI

$10.5151 / 9788580391282-11$

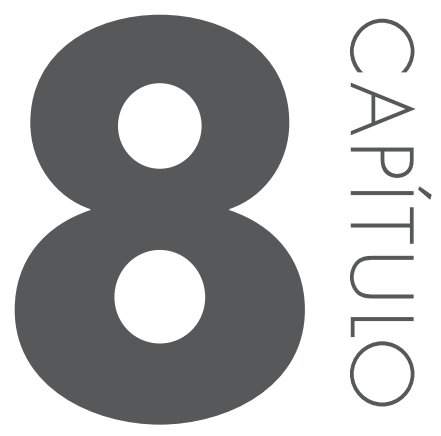

\title{
DESENHO UNIVERSAL PARA APRENDIZAGEM E MOOCs: UMA REFLEXÃO PRELIMINAR
}

Solange Cristina da SILVA; Marcio Vieira de SOUZA

\section{INTRODUC̣ÃO}

$\mathrm{Na}$ sociedade atual, entendida como sociedade do conhecimento, é premissa consensual garantida por lei que as pessoas com deficiência tenham acessibilidade em todos os âmbitos. Deste modo, a inclusão mais do que um discurso, deve ser construída como cultura social. Assim sendo, os vários espaços, sejam eles de lazer, de trabalho ou de aprendizagem devem oportunizar o acesso a todos. $\mathrm{Na}$ esfera educacional, além da acessibilidade arquitetônica, devem ser disponibilizados recursos que possam facilitar o acesso ao conhecimento e o processo educativo de forma igualitária.

$\mathrm{Na}$ educação a distância não é diferente. Muitas universidades brasileiras, atualmente, são conveniadas com a Universidade Aberta do Brasil, para oferecer 
cursos nessa modalidade. A maioria delas utilizada, para o desenvolvimento do processo ensino-aprendizagem, o ambiente virtual de aprendizagem Moodle. Esse ambiente é o espaço principal de interação entre o professor e os estudantes e nele, está consta todo o conteúdo a ser trabalhado no curso. Assim sendo, esse ambiente tem que ser acessível tanto na sua estrutura, como na organização, possibilitando a acessibilidade, usabilidade e flexibilidade.

Ainda, nessa modalidade de educação, várias Universidade, tanto nacionais como internacionais, utilizam os MOOCs. Da mesma forma, o ambiente educativo dos MOOCs deve ser acessível a todos, considerando a diversidade de pessoas que possam acessá-las.

A modalidade de educação a distância atende a uma demanda cada vez maior de estudantes, não só da graduação, mas de diversos outros cursos como extensão e pós-graduação. Nesse contexto, o público que acessa esses ambientes educativos é diverso, com características, necessidades e potencialidades diferentes. Para tanto, é preciso cada vez mais desenvolver recursos cada vez mais acessíveis e universais. Assim sendo, buscamos neste texto refletir sobre a contribuição do Desenho Universal para Aprendizagem na construção de ambientes virtuais de aprendizagem como os citados anteriormente.

Em 2012 e 2013 foi realizada uma pesquisa intitulada Ambiente Virtual de Aprendizagem Moodle: acessibilidade nos processos de aprendizagem na Educação a Distância/CEAD/UDESC (SILVA et al., 2013) articulada ao LEdI - Laboratório de Educação Inclusiva/CEAD/UDESC, com o objetivo de analisar a acessibilidade dos conteúdos postados no ambiente virtual de aprendizagem Moodle de duas disciplinas utilizado no Curso de Pedagogia a Distância do CEAD/UDESC, propondo alternativas de solução visando a construção de um ambiente de aprendizagem acessível a todos/as discentes. Considerando esses aspectos, a partir dessa pesquisa iniciamos uma reflexão sobre a contribuição do Desenho Universal para Aprendizagem voltadas para os espaços MOOCs.

\section{A EDUCAC̣ÃO EM REDE E A FORMAC̣ÃO DOS EDUCADORES}

No mundo contemporâneo estamos vivenciando inúmeras transformações pertinentes à globalização. As transformações tecnológicas, hoje, são de tamanha magnitude, que praticamente todos os países do mundo tem acesso "a rede". Em nível social e político, a sociedade contemporânea tem trabalhado o conceito de rede em várias esferas e contextos. 
Atualmente, na era da informação e do conhecimento: a economia, a sociedade e a cultura estão sendo estudadas como uma sociedade em rede (CASTELLS, 1999). Sabe-se que a globalização nos apresenta relações antagônicas, com benefícios (acessibilidade, rapidez nas informações, ligação com o mundo, novos meios de estudo e capacitação) e malefícios (alienação, exclusão social e ritmo acelerado), mas sabemos que os benefícios não são pequenos, e para que se faça um enfretamento, necessita-se de políticas públicas eficazes, particularmente na área da educação. Transformações relevantes estão ocorrendo em diversas áreas do conhecimento. Um especial impulso vem se desenvolvendo nos sistemas de comunicação com grande velocidade, e a partir desta premissa é que novas demandas emergem na sociedade brasileira (MARTINS; SOUZA, 2009). Atrelada as mais significativas transformações, está a Educação a Distância e sua viabilização como política pública.

Nesse sentido, é necessário que se viabilizem políticas públicas de $\mathrm{EaD}$ voltadas para a formação dos professores a fim de atender as expectativas de uma formação acadêmica de qualidade no âmbito do cenário educacional brasileiro em uma época em que os estudantes são nativos digitais em sua grande maioria. Neste rumo está a Educação a Distância e seus desafios, como por exemplo, incluir a acessibilidade e a usabilidade nas ferramentas de apoio a EaD, como no caso dos AVA.

Mesmo sabendo-se das carências, atualmente já se busca agregar redes sociais aos mais variados AVA, como por exemplo, o uso de microblogging, de software social, de redes sociais virtuais privadas como AVA, entre outras iniciativas. Obviamente que as ferramentas tecnológicas, no caso os AVA, não suprem todas as lacunas educacionais, pois se constata que também faltam metodologias e técnicas pedagógicas pensadas para o uso desses suportes.

Concomitantemente, acompanhando as mudanças tecnológicas e sociais estão surgindo novas plataformas educacionais com cursos com características massivas, novos AVA para serem utilizados totalmente pela internet e com mais autonomia pelos estudantes, os MOOCs (SOUZA, 2013). De acordo com Tavares (2014, p. 30),

Os MOOCs se dividem ainda em duas categorias distintas: os cMOOC e o xMOOC. O primeiro grupo é baseado no Conectivismo e se desenvolvem de maneira mais informal e são independentes de uma instituição tradicional de ensino. Os cMOOCs atrai as massas, pois os estudantes são encorajados a irem mais além em busca de mais conhecimentos na própria rede. Ou seja, utilizam a ferramenta que tem em mãos para ampliar o aprendizado por meio da pesquisa 
e busca por mais informações, aumentando a interatividade entre os participantes nas diferentes plataformas tecnológicas. $\mathrm{O}$ segundo grupo, os xMOOCs, condizem com a extensão dos modelos pedagógicos das universidades no ciberespaço. Isto é, os formatos são pré-definidos pelos professores da universidade, onde a figura do professor é central e a interatividades entre os estudantes não é prioridade. Em suma, tem-se a duplicidade do conhecimento

Assim, os MOOCs podem ser uma alternativa para responder a demanda de formação de professores, oportunizando diálogos interdisciplinares, maior autonomia, um conhecimento aberto e cooperação. Por outro lado, essa ferramenta educacional traz novos desafios, dos quais destacamos a questão da acessibilidade. Com o intuito de refletirmos sobre esse aspecto trazemos a contribuição do Desenho Universal para Aprendizagem.

\section{DESENHO UNIVERSAL PARA APRENDIZAGEM: CONTRIBUINDO PARA A EDUCAC̣ÃO A DISTÂNCIA}

Refletir sobre uma educação para todos na Educação a Distância $(\mathrm{EaD})$ vai ao encontro da premissa da Educação Inclusiva, que está fundamentada na concepção de direitos humanos e visa garantir a todos/as igualdade de oportunidades de aprendizagem e não somente as pessoas com deficiência. Como argumenta Miranda (2002),

[...] há muitas situações em que as tecnologias que dão suporte a essa modalidade de ensino não são acessíveis para todas as pessoas, principalmente aquelas com alguma deficiência específica. A exemplo, pode-se citar as páginas web que usam imagens sem equivalentes textuais. Páginas com essas características impedem que usuários com limitações visuais tenham acesso a informação de forma completa. Ainda, falando de imagem, outro exemplo que impede a acessibilidade em cursos a distância via web é quando os equipamentos utilizados têm muito baixa resolução, o que impede, igualmente, o acesso à informação. (p.18)

Principalmente na EaD, é imprescindível que os recursos tecnológicos disponíveis sejam qualitativamente acessíveis, não como forma de concessão aos grupos, mas como direito de todos/as. 
Os recursos de Tecnologia Assistiva são fundamentais para que haja acessibilidade as informações disponíveis no meio digital aos usuários com deficiência. De acordo com o Comitê de Ajudas Técnicas da Coordenadoria Nacional da Pessoa Portadora de Deficiência, a Tecnologia Assistiva é uma área do conhecimento, interdisciplinar, que engloba produtos, recursos, metodologias, estratégias, práticas e serviços, que promovam a funcionalidade das pessoas com deficiência, incapacidades ou mobilidade reduzida, objetivando sua autonomia, independência, qualidade de vida e inclusão social (ITS Brasil, 2008, p. 10).

De acordo com o Art. $8^{\circ}$, do Decreto 5296 (2004), acessibilidade é a [...] condição para utilização, com segurança e autonomia, total ou assistida, dos espaços, mobiliários e equipamentos urbanos, das edificações, dos serviços de transporte e dos dispositivos, sistemas e meios de comunicação e informação, por pessoa portadora de deficiência ou com mobilidade reduzida.

O consultor Romeu Kazumi Sassaki (apud VIVARTA, 2003, p. 24-25) esclarece que para uma sociedade ser acessível é preciso que ela contemple: acessibilidade arquitetônica; acessibilidade comunicacional; Acessibilidade metodológica; acessibilidade instrumental; acessibilidade programática e; acessibilidade atitudinal.

Considerando esses aspectos, para tornar os ambientes virtuais acessíveis a todos, temos que considerar as diversas características dos participantes, seu perfil, seu ritmo e forma de aprendizagem.

Dentro dessa premissa, conforme mencionado anteriormente realizamos uma pesquisa teórica, que teve como teoria principal o Desenho Universal para a Aprendizagem, com o propósito de contribuir na organização do ambiente virtual de aprendizagem nos cursos a distância disponibilizados por meio do Moodle.

O termo Universal Design surgiu na Arquitetura e influenciou a mudança de paradigma no desenvolvimento de projetos urbanos, de arquitetura e design, inclusive de produtos. Assim, o conceito de Desenho Universal surgiu em decorrência de reivindicações de dois segmentos sociais. O primeiro composto por pessoas com deficiência que não sentiam suas necessidades contempladas nos espaços projetados e construídos. O segundo formado por arquitetos, engenheiros, urbanistas e designers que desejavam maior democratização do uso dos espaços e tinham uma visão mais abrangente da atividade projetual. (GOVERNO DO ESTADO DE SÃO PAULO, 2010, p.14)

Este conceito, também conhecido como Design Universal relaciona-se ao conceito de "Design Inclusivo" e de "Design para todos". A premissa principal deste conceito refere-se ao fato de que os ambientes ou produtos sejam 
desenvolvidos para ser usado por todas as pessoas, na sua máxima extensão possível, não necessitando de adaptações para atender as especificidades. (Governo do Estado de São Paulo, 2010)

Com isso, ele propõe soluções que compõe o escopo da acessibilidade para que haja a participação efetiva e a utilização dos recursos e/ou produtos por todos, independentemente da idade, tamanho, condição sensorial ou física, bem como qualquer outra característica que possa ter. Para tanto, apresenta como princípios básicos: equiparação nas possibilidades de uso; flexibilidade no uso; uso simples e intuitivo; captação da informação; tolerância ao erro; mínimo esforço; dimensão e espaço para uso e interação (CENTRO DE ESTUDOS E PESQUISA MUNICIPAL - CEPAM, 2008, 52-53).

Segundo Campos \& Mello (s/d, p. 02), o Desenho Universal quando transposto para o contexto da Educação, é um conjunto de princípios para o desenvolvimento de ambientes e recursos pedagógicos que possibilitam processos de ensino e de aprendizagem ao maior número de pessoas; onde devemos pensar em alternativas, diferentes formas de acesso ao conteúdo pedagógico, diferentes formas de participação, estilos de aprendizagem, habilidades e deficiências, além de variados contextos de aprendizagem.

Assim, o Desenho Universal para Aprendizagem (Universal Design for Learning - UDL), traz referências para que se possa ultrapassar as barreiras no processo ensino-aprendizagem e os currículos rígidos, propondo um modelo para todos. Nesse sentido, a proposta trazida pela UDL é que se construam espaços educativos flexíveis, com a apoio de recursos e materiais, para o uso de todos/as contemplando diferentes formas de aprender e diferentes ritmos de aprendizagem.

De acordo com Center for Applied Special Technology - CAST (2013), no Desenho Universal para Aprendizagem devemos considerar as três redes cerebrais primárias envolvidas no processo de aprendizagem, as quais cada uma está ligada a um princípio da UDL: a) Redes de conhecimento: ligada ao "o quê" da aprendizagem; o como reunimos fatos e categorizamos o que vemos, ouvimos e lemos. Está relacionada com o primeiro princípio do DU, da representação; b) Redes estratégicas: relacionadas ao "como" da aprendizagem, refere-se ao como planejamos e executamos as tarefas, bem como nos organizamos e expressamos nossas ideias. Está relacionada o segundo princípio do DU, da ação e expressão; c) Redes afetivas: relacionadas ao "porquê" da aprendizagem, no sentido de como ficamos motivados, interessados e somos desafiados para aprendizagem. Está ligada ao terceiro princípio do DU, do engajamento.

Levando em conta todos esses aspectos, concluímos que esta teoria apresenta conceitos e normas que contribuem para a composição do ambiente virtual e a forma de apresentação dos conteúdos postados com vista a acessibilidade. 
$\mathrm{Na}$ análise dos dados de como foram postados os conteúdos e a organização do ambiente virtual Moodle de duas disciplinas do Curso de Pedagogia a Distância, a partir das categorias do Desenho Universal para Aprendizagem, na pesquisa anteriormente mencionada, constatamos (SILVA et al., 2013, p. 10-11):

Em relação a categoria 1, evidenciou-se que, apesar do uso de vários recursos visuais e auditivos para apresentação do conteúdo, os mesmos tinham conteúdos diferenciados e os estes conteúdos foram apresentados mais especificamente para pessoas videntes e ouvintes. Na pesquisa, constatou-se que apenas uma disciplina tinha descrição das imagens, porém não havia em ambas legendas nos vídeos e opção de LIBRAS para os mesmos e para o conteúdo apresentado em forma de textos escritos. Os textos postados eram na maioria extensos, não contemplando assim as pessoas com dislexia ou com dificuldade na leitura.

$\mathrm{Na}$ categoria 2, constatou-se que no ambiente virtual de aprendizagem Moodle, e a dos estudantes, o qual era a escrita. Sendo assim, não foi possibilitado outra forma de resposta, como por exemplo, em áudio ou em vídeo. Assim, os docentes surdos ou que tivessem maior facilidade na comunicação visual, teriam maior dificuldade para expressar seus conhecimentos pela escrita, bem como ficavam impedidos de compreender o conteúdo dos materiais postados em áudio.

$\mathrm{Na}$ terceira e última categoria, percebeu-se que o fórum era um recurso usado também para motivação e engajamento. Porém, este aspecto não foi suficientemente analisado, no sentido de que o objeto a ser pesquisado eram os conteúdos postados e não a relação professor-estudante. Visto que, as disciplinas foram pesquisadas foram analisadas de forma estática, na em seu processo de desenvolvimento.

Sendo assim, considerando que os estudantes diferem marcadamente nas formas em que são motivados a aprender e o que os estimulam para seu engajamento, este aspecto não foi identificado com relevância. Outro ponto, constatado foi que as tarefas eram propostas para serem desenvolvidas algumas em grupo e outras sozinhas. Entretanto, pela pesquisa não foi percebida o fornecendo de múltiplas opções para o engajamento dos estudantes. 
$\mathrm{Na}$ análise dos dados pudemos constatar que nenhuma dessas categorias estava suficientemente contemplada nos ambientes pesquisados, o que, certamente dificulta o acesso igualitário a todos os estudantes.

Mesmo essa pesquisa estando voltada para a análise do ambiente virtual de aprendizagem moodle, seus resultados nos dão pistas para pensarmos também a acessibilidade nos MOOCs.

$\mathrm{O}$ ambiente virtual destinado a aprendizagem, possui diferentes modelos, e deve possibilitar a universalização do acesso ao conteúdo dos cursos oferecidos aos diferentes perfis de usuários, podendo assim tornar-se efetivamente inclusivo.

Ao analisarmos a acessibilidade sobre os conteúdos postados no ambiente virtual de aprendizagem Moodle, constatamos que este ambiente traz aspectos de acessibilidade na sua estrutura. Porém, considerando os diferentes perfis de aprendizagem, a maneira como o conteúdo é inserido neste ambiente virtual pode tornar-se uma barreira para a aprendizagem.

Concluímos com isso, que o Desenho Universal para Aprendizagem nos aponta para a necessidade de flexibilidade e personalização do próprio estudantes para as formas de apresentação do conteúdo e a expressão do conhecimento que melhor se adéqua a seu perfil de aprendizagem. Para tanto, é imprescindível que, o mesmo conteúdo, seja apresentado de diferentes formas e o ambiente de aprendizagem, seja ele qual for, oportunize a flexibilização do conteúdo, para que este possa ser acessado com mais facilidade e possibilitar um aprendizado verdadeiramente significativo e inclusivo. Sendo assim, no ambiente virtual de aprendizagem para todos, é ainda uma meta a ser conquistada. 


\section{REFERÊNCIAS}

CAMPOS, Talita de; MELLO, Maria Aparecida Ferreira de. 0 Desenho Universal e a Tecnologia Assistiva como Potencializadores dos Processos de Ensino e Aprendizagem Parte II. MEDICINA DE REABILITAÇÃO, v. 93.

CASTELLS, Manuel. Fim do milênio: a era da informação: economia, sociedade e cultura. In: Redes informatizadas de comunicação: a teia de rede internacional DPH /Márcio Vieira de Souza - São Paulo: Blucher Acadêmico, 2008.

Center for Applied Special Technology. About UDL. 20013. Disponível em: http://www.cast.org/udl/ index.html. Acesso: 20 abril 2013.

Center for Applied Special Technology. UDL Guidelines Version 2.0. 2002. Disponível: de http://www. udlcenter.org/aboutudl/udlguidelines. Acesso: 20 abril 2012.

CENTRO DE ESTUDOS E PESQUISA MUNICIPAL. Fundação Prefeito Faria Lima. Acessibilidade nos municípios: como aplicar o decreto 5296/04. 2008. Disponível em: http://www.cepam.sp.gov.br/ arquivos/acessibilidade/Acessibilidade_nos_municipios.pdf. Acesso: 05 de maio 2012.

BRASIL. DECRETO n 5296 de 02 de dezembro de 2004. Brasilia, 2004. Disponível em: http://www. planalto.gov.br/ccivil_03/_ato2004-2006/2004/decreto/d5296.htm. Acesso: 10 abril 2012.

DOTTA, S. Uso de uma mídia social como ambiente virtual de aprendizagem. Anais do XXII SBIE - XVII WIE. Aracaju: SBC. 2011. p. 610-619.

FREIRE, P. Pedagogia do oprimido. 17ª Ed. Rio de Janeiro: Paz e Terra, 1987.

GALVÃO FILHO, T. A. A Tecnologia Assistiva: de que se trata? In: MACHADO, G.

GOMES, M. V. Educação em rede: uma visão emancipadora. São Paulo: Editora Cortez, 2004.

GOVERNO DO ESTADO DE SÃO PAULO. Desenho universal: Habitaç̃̃o de interesse social no Estado de São Paulo. 2010. Disponivel em: http://www.mpsp.mp.br/portal/page/portal/Cartilhas/manualdesenho-universal.pdf. Acesso em 05 maio de 2013.

ITS BRASIL. Tecnologia Assistiva nas Escolas: Recursos básicos de acessibilidade sócio-digital para pessoas com deficiência. Disponível em: http://itsbrasil.org.br/publicacoes/cartilha/cartilha-tecnologiaassistiva-nas-escolas-recursos-basicos-de-acessibilidade. Acesso: 03 janeiro 2012.

MACIEL, I. M. Educação a Distância: Ambiente virtual, construindo significados. Disponível em: http:// www.senac.br/BTS/283/boltec283e.htm. Acesso em 02 ago. 2012.

MARTINS, Gisele; SOUZA Marcio Vieira de. Educação a Distância como política pública no Brasil. SP: ABED, 2009. Disponivel em: http://www.abed.org.br/congress02009/CD/trabalhos/1552009155011. pdf. Acesso em 22 mar 2015.

MIRANDA, A. S. Recomendações de acessibilidade digital em cursos de educação a distância via web para portadores de deficiência visual. Dissertação de Mestrado. Universidade Federal de Santa Catarina, Florianópolis, SC, Brasil, 2002.

PRIMO, A. 0 aspecto relacional das interações na Web 2.0. XXIX Congresso Brasileiro de Ciências da Comunicação. Brasilia: Sociedade Brasileira de Estudos Interdisciplinares da Comunicaccão, 2006.

SERRÃO, T. et al. Construção Automática de Redes Sociais Online no Ambiente Moodle. Anais do XXII 
SBIE - XVII WIE. Aracaju: SBC, 2011. p. 924-933

SILVA, S.C. da; SOUZA, M. V. de; BECHE, R.C. E.; BOCK, G. L. K. Educação a Distância e Acessibilidade: uma parceria indispensável. Anais do IV Congresso Internacional do Conhecimento e Inovação - CIKI, 2014. Disponível em: http://www.egc.ufsc.br/ciki/edicoes_anteriores. Acesso: 09 junho 2015.

SILVA, S.C.; BOCK, G. L. K.; BECHE, R. C. E.; GOEDERT, L. Ambiente Virtual de Aprendizagem Moodle: Acessibilidade Nos Processos De Aprendizagem Na Educação a Distância/CEAD/UDESC. Anais do X Congresso Brasileiro de Ensino Superior a Distância - ESUD, 2013. Disponível em: http://www.aedi. ufpa.br/esud/trabalhos/oral/AT4/114280.pdf. Acesso: 05 jun 2015.

SOUZA, M.V. et al. MÍDIAS SOCIAIS, AVA e MOOCs: REFLEXÕES SOBRE EDUCAÇ̃̃O EM REDE. ICBL 2013: - International Conference on Interactive Computer aided Blended Learning, Florianópolis, v. n., p.183-190, nov. 2013. Disponivel em: <http://www.icbl-conference.org/proceedings/2013/ papers/Contribution62_a.pdf>. Acesso em: 12 mar. 2014.

SOUZA, M.V. Redes informatizadas de comunicação: a teia da rede internacional DPH. São Paulo: Blucher Acadêmico, 2008.

SOUZA, Marcio Vieira; SIMON, Rangel Machado. Redes sociais virtuais e novas formas de aprendizado: reflexões sobre educação em rede. In: Coleção Mídias Contemporâneas: possibilidades e desafios. Vol. I. SOUZA, Carlos Alberto de; MORALES, Ofélia (orgs.). PG: PROEX/UEPG, 2014. Disponível em: <http:// vepgfocafoto.wordpress.com>. Acesso em 23 de mar de 2015.

TAVARES, V. B. A. Massive Open Online Courses (M00CS): Nova tendência educacional. 2014. Disponível em: <http://bdm.unb.br/bitstream/10483/8387/1/2014_VivianeBrunellyTavares.pdf>. Acesso em: 01 jul 2015.

TEIXEIRA, E; MEDEIROS, F. P. A. D; GOMES, A. S. Microblogging como estilo de interação e colaboração em Ambientes Virtuais de Ensino e Aprendizagem. Anais do XXII SBIE - XVII WIE. Aracaju: SBC, 2011. p. 956-959.

VIVARTA, V. Mídia e deficiência. 2003. Disponível em: <http://www.andi.org.br/sites/default/files/ Midia_e_deficiencia.pdf>. Acesso: 10 jan 2014. 